Supporting information for:

\title{
Mercury methylating microbial community structure in boreal wetlands explained by local physicochemical conditions
}

Jingying Xu${ }^{1}$, Van Liem-Nguyen ${ }^{2}$, Moritz Buck ${ }^{1,3}$, Stefan Bertilsson ${ }^{1,3}$, Erik Björn ${ }^{2}$, Andrea G. Bravo ${ }^{1,4 *}$

${ }^{1}$ Department of Ecology and Genetics, Limnology, Uppsala University, Uppsala, Sweden

${ }^{2}$ Faculty of Science and Technology, Department of Chemistry, Umeå University, Sweden

${ }^{4}$ Swedish University of Agricultural Sciences (SLU), Sweden

${ }^{4}$ Institute of Marine Sciences (ICM), Spanish National Research Council (CSIC), Spain

*Andrea G. Bravo: jandriugarcia@gmail.com 
Figure S1. Rarefaction curve of the $h g c A$ gene. Each line represents a wetland soil sample and is coloured by the site it was collected (blue: SKM; dark brown: LDN, orange: GTN; purple: KSN).

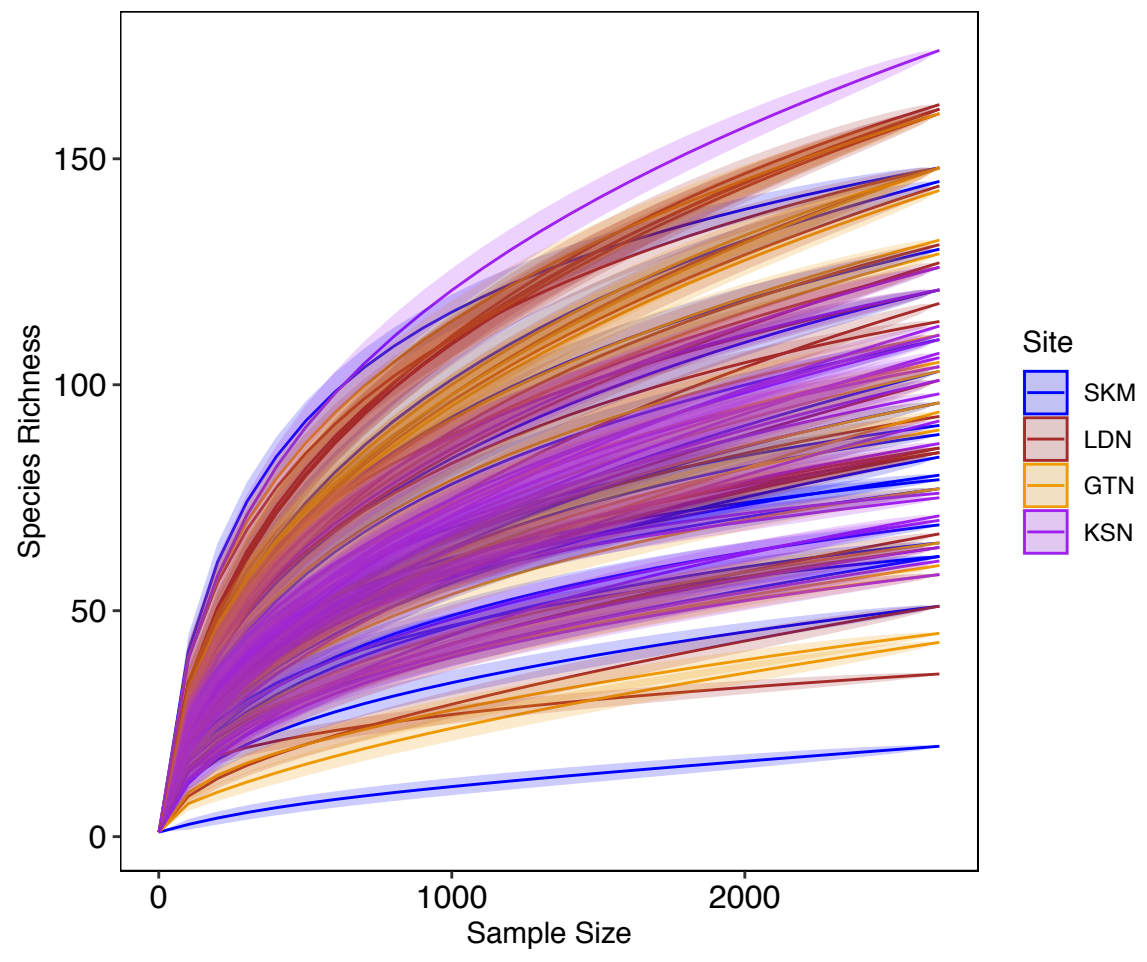

Figure S2. Nonmetric multidimensional scaling (NMDS) ordination of $\mathrm{Hg}^{\mathrm{II}}$ methylating microbial communities based on the Bray-Curtis dissimilarity of community composition at SKM (A), KSN (B), LDN (C) and GTN (D). Sites within a wetland are color coded and core-depth is shaped-coded.
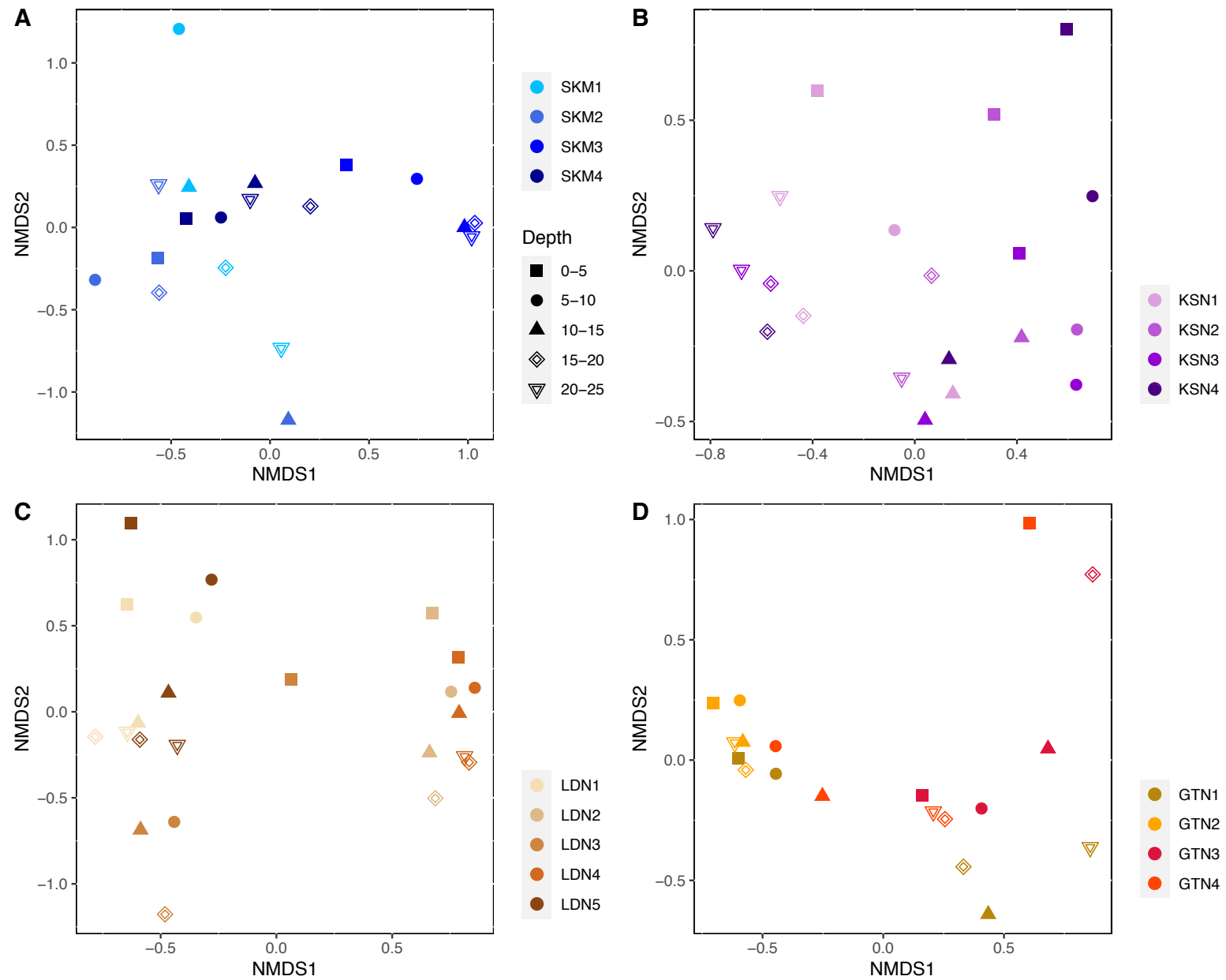
Figure S3. Significant $(p<0.05)$ spearman correlations between different physicochemical parameters. The ellipses have their eccentricity parametrically scaled to the Pearson correlation value (narrower ellipses represents higher correlation values). The orientation of the ellipse indicates negative (red) or positive (blue) correlations (scale on the right).

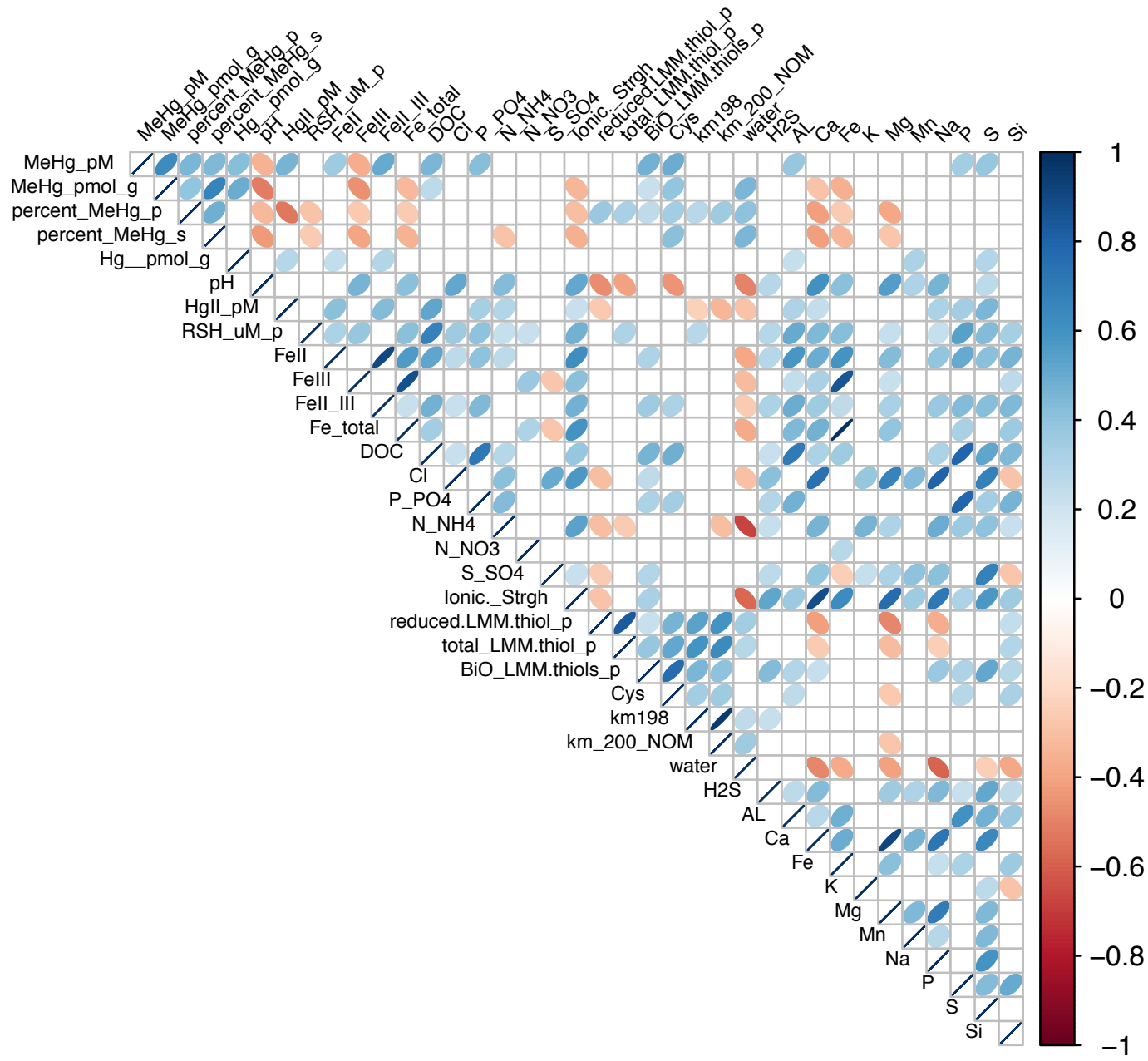


Figure S4. Heat map of Spearman significant $(\mathrm{p}<0.05)$ correlations between environmental variables and $h g c A+$ taxa at order level detected in the studied wetland soils. Colors represent the significant p-values of Spearman correlations. Red stands for negative significant correlations and blue for positive and significant correlations (scale on the right).

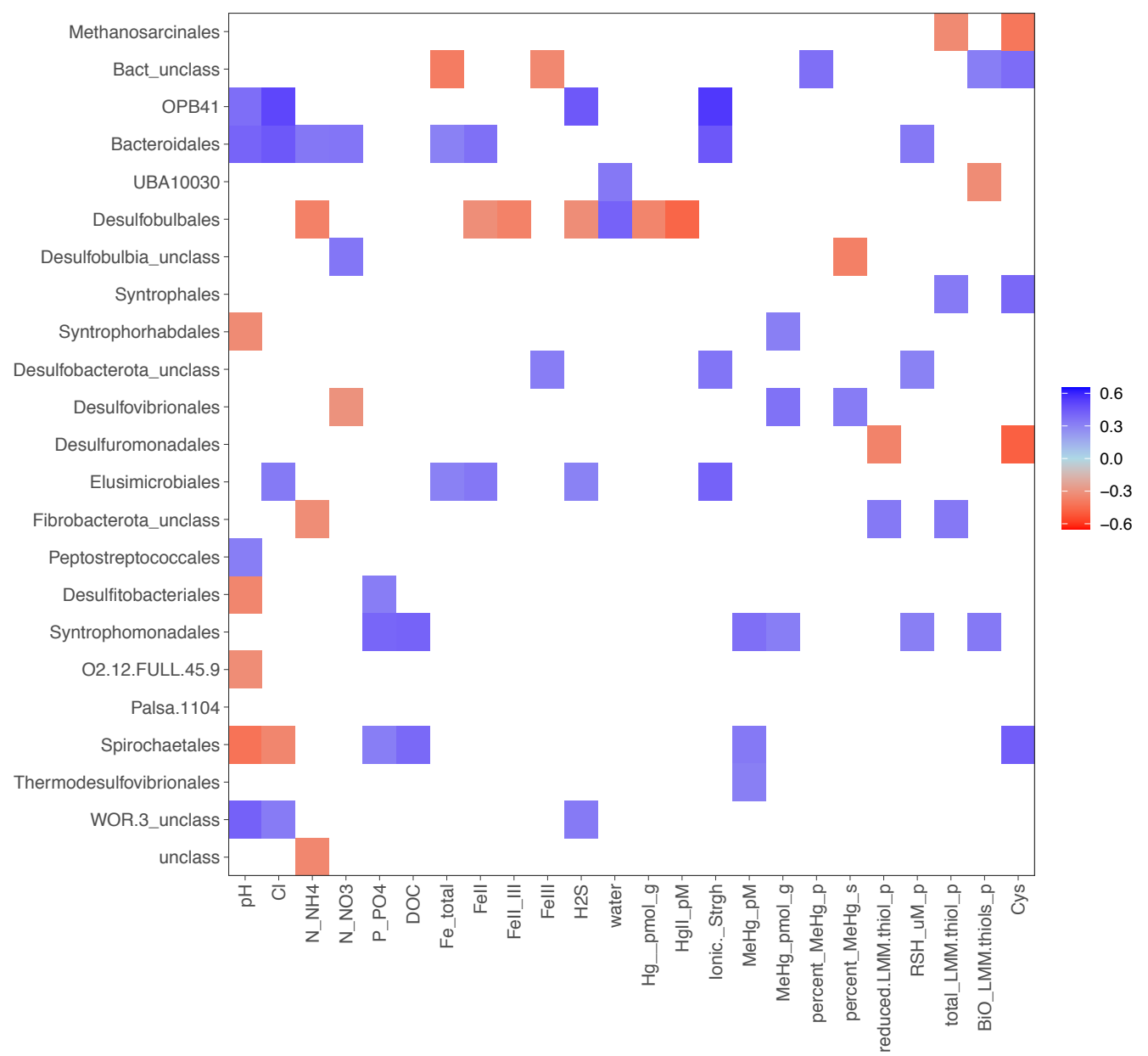

Figure S5. Left panel: Correlation plot (Mantel $\mathrm{R}=0.13, \mathrm{p}$-value $=0.003)$ between $h g c A+$ microbial community (Bray Curtis) dissimilarity and the Euclidean distance of $k_{\mathrm{m}}\left(k_{\mathrm{m}}\right.$ of spiked inorganic ${ }^{200} \mathrm{Hg}$ bound to organic matter). Right panel: Regression analyses between the second component of the Principal coordinate analysis performed with the hgcA + microbial community and $k_{\mathrm{m}}\left(k_{\mathrm{m}}\right.$ of spiked inorganic- ${ }^{200} \mathrm{Hg}$ bound to organic matter).
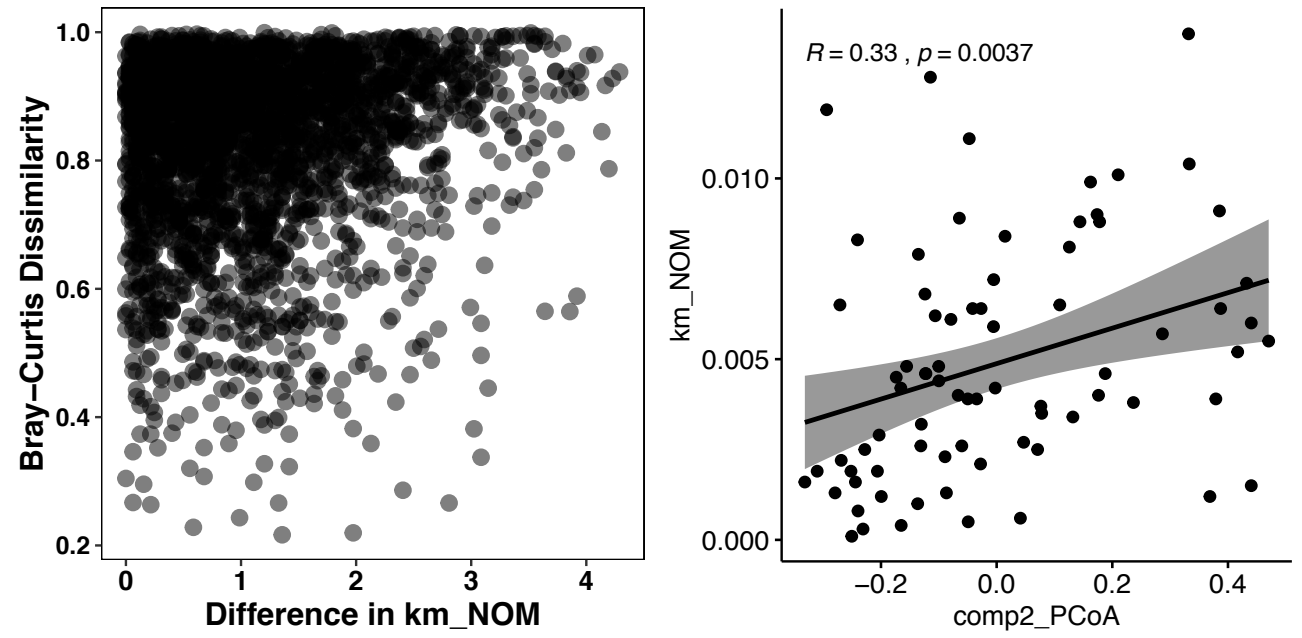
Figure S6. Relative abundance (\%) of $h g c A+$ Geopsychrobacteraceae family at the different studied wetlands.

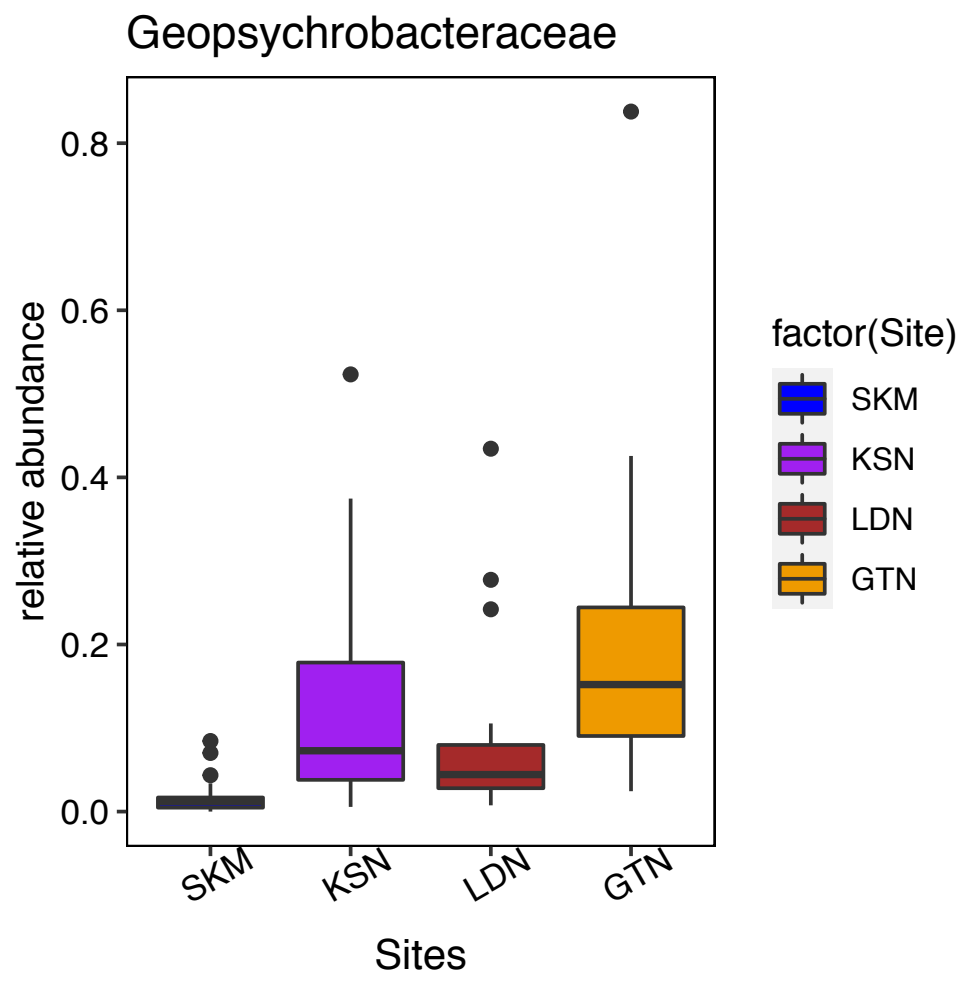

Table S1. Characterisation of the boreal wetlands. SKM: Storkälsmyran, KSN: Kroksjön, LDN: Långedalen and GTN: Gästern. All data presented in this table were extracted from Liem et al., (2020).

\begin{tabular}{|c|c|c|c|c|c|c|c|c|c|}
\hline Location & Depth & $\begin{array}{c}\mathbf{H g}^{\mathrm{II}} \\
\mathrm{pmol} \mathrm{g}^{-1}\end{array}$ & $\begin{array}{c}\text { MeHg } \\
\text { pmol g-1 }^{-1}\end{array}$ & 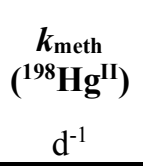 & $\begin{array}{c}k_{\text {meth }} \\
\left({ }^{200} \mathbf{H g}^{\mathrm{II}}-\right. \\
\text { NOM }) \\
\mathrm{d}^{-1} \\
\end{array}$ & $\begin{array}{c}\text { Dry } \\
\text { weight } \\
\% \\
\%\end{array}$ & $\begin{array}{l}\text { DOC } \\
\mathrm{mg} / \mathrm{L}\end{array}$ & $\begin{array}{l}\mathrm{P}^{-\mathrm{PO}_{4}} \\
\mu \mathrm{g} \mathrm{L}^{-1}\end{array}$ & $\mathrm{~N}-\mathrm{NH}_{4}$ \\
\hline SKM-1 & $0-5$ & 600 & 41.4 & 0.0204 & 0.006 & 10.7 & 60.6 & 6.6 & 100 \\
\hline SKM-1 & $5-10$ & 695 & 45.7 & 0.0442 & 0.0119 & 9.26 & 63.41 & 1.2 & 10.1 \\
\hline SKM-1 & $10-15$ & 491 & 39 & 0.0384 & 0.0083 & 13.1 & 68.43 & 2.1 & 15.5 \\
\hline SKM-1 & $15-20$ & 405 & 41 & 0.0096 & 0.0019 & 15.3 & 66.87 & 10 & 19.3 \\
\hline SKM-1 & $20-25$ & 452 & 41.8 & 0.0062 & 0.0019 & 14.4 & 68.03 & 18 & 41.2 \\
\hline SKM-2 & $0-5$ & 1010 & 93.9 & 0.0293 & 0.0065 & 11.9 & 66.85 & 51 & 597 \\
\hline SKM-2 & $5-10$ & 752 & 97.4 & 0.012 & 0.0022 & 13.7 & 68.22 & 145 & 1490 \\
\hline SKM-2 & $10-15$ & 607 & 48.5 & 0.0091 & 0.0016 & 14.5 & 66.61 & 111 & 1080 \\
\hline SKM-2 & $15-20$ & 499 & 30 & 0.0083 & 0.0016 & 15.8 & 73.97 & 194 & 1550 \\
\hline SKM-2 & $20-25$ & 552 & 47.2 & 0.014 & 0.0029 & 12.2 & 72.56 & 131 & 1190 \\
\hline SKM-3 & $0-5$ & 501 & 53.3 & 0.0201 & 0.0057 & 18.1 & 68.82 & 12 & 58.6 \\
\hline SKM-3 & $5-10$ & 619 & 87.5 & 0.0209 & 0.0037 & 17.2 & 70.07 & 2.8 & 40.3 \\
\hline SKM-3 & $10-15$ & 608 & 24.4 & 0.0379 & 0.0062 & 17.5 & 52.04 & 2.6 & 33.1 \\
\hline SKM-3 & $15-20$ & 584 & 13.7 & 0.0374 & 0.0068 & 17.7 & 53.49 & 6 & 37.3 \\
\hline SKM-4 & $0-5$ & 652 & 39.2 & 0.0202 & 0.0046 & 15.9 & 71.02 & 5.4 & 27.3 \\
\hline SKM-4 & $5-10$ & 659 & 50.2 & 0.0367 & 0.0088 & 15.6 & 68.3 & 2.5 & 12.9 \\
\hline SKM-4 & $10-15$ & 424 & 44.4 & 0.0486 & 0.0088 & 18.6 & 88.81 & 17 & 110 \\
\hline SKM-4 & $15-20$ & 360 & 40.7 & 0.0329 & 0.0059 & 16.8 & 85.41 & 26 & 137 \\
\hline SKM-4 & $20-25$ & 349 & 15.5 & 0.0303 & 0.0065 & 18.9 & 84.72 & 39 & 397 \\
\hline LDN-1 & $0-5$ & 945 & 92 & 0.0182 & 0.0046 & 6.11 & 67.4 & 1.2 & 22.9 \\
\hline LDN-1 & $5-10$ & 1290 & 133 & 0.0327 & 0.0048 & 6.41 & 71.38 & 5.3 & 9.06 \\
\hline LDN-1 & $10-15$ & 818 & 135 & 0.0568 & 0.0101 & 4.31 & 77.55 & 9.7 & 7.92 \\
\hline LDN-1 & $15-20$ & 1260 & 163 & 0.0351 & 0.0064 & 5.97 & 62.17 & 13 & 22.4 \\
\hline LDN-1 & $20-25$ & 1500 & 132 & 0.0169 & 0.0042 & 6.62 & 55.88 & 14 & 57.6 \\
\hline LDN-2 & $0-5$ & 88.8 & 13.4 & 0.001 & 0.0001 & 19.7 & 44.93 & 1 & 90.6 \\
\hline LDN-2 & $5-10$ & 353 & 13.7 & 0.0019 & 0.0003 & 27.8 & 39.45 & 2 & 121 \\
\hline LDN-2 & $10-15$ & 225 & 6.11 & 0.0062 & 0.001 & 44 & 38.92 & 1.8 & 150 \\
\hline LDN-2 & $15-20$ & 115 & 2.79 & 0.0038 & 0.0004 & 61.4 & 39.59 & 3.1 & 432 \\
\hline
\end{tabular}




\begin{tabular}{|c|c|c|c|c|c|c|c|c|c|}
\hline LDN-3 & $10-15$ & 638 & 50.6 & 0.0212 & 0.0038 & 8.12 & 35.88 & 1.8 & 3.6 \\
\hline LDN-3 & $15-20$ & 969 & 17.5 & 0.0043 & 0.0006 & 6.88 & 33.88 & 3.4 & 14.7 \\
\hline LDN-4 & $0-5$ & 822 & 45.9 & 0.0087 & 0.0012 & 19.1 & 19.3 & 0.6 & 144 \\
\hline LDN-4 & $5-10$ & 876 & 54.7 & 0.0138 & 0.0019 & 20.5 & 18 & 0.8 & 167 \\
\hline LDN-4 & $10-15$ & 731 & 28 & 0.0079 & 0.0013 & 23.6 & 17.77 & 1 & 629 \\
\hline LDN-4 & $15-20$ & 497 & 12.5 & 0.0129 & 0.0025 & 33.9 & 28.09 & 1 & 4490 \\
\hline LDN-4 & $20-25$ & 687 & 68.8 & 0.0044 & 0.0008 & 42.2 & 26.44 & 1.1 & 4120 \\
\hline LDN-5 & $0-5$ & 998 & 70.7 & 0.002 & 0.0005 & 3.11 & 80.75 & 5.4 & 32.7 \\
\hline LDN-5 & $5-10$ & 1120 & 94.2 & 0.0106 & 0.0021 & 2.7 & 89.46 & 4.9 & 10.9 \\
\hline LDN-5 & $10-15$ & 1200 & 72.8 & 0.005 & 0.0012 & 5.47 & 93.86 & 7.3 & 50.1 \\
\hline LDN-5 & $15-20$ & 1020 & 55.2 & 0.0271 & 0.0039 & 12.9 & 145 & 18 & 47.5 \\
\hline LDN-5 & $20-25$ & 567 & 24 & 0.0105 & 0.0015 & 13.5 & 235.6 & 23 & 64.2 \\
\hline GTN-1 & $0-5$ & 422 & 11.1 & 0.0338 & 0.0045 & 11.6 & 49.12 & 6.2 & 1070 \\
\hline GTN-1 & $5-10$ & 453 & 6.44 & 0.0189 & 0.004 & 23.7 & 57.5 & 3.7 & 1770 \\
\hline GTN-1 & $10-15$ & 371 & 2.96 & 0.0404 & 0.0071 & 25.4 & 61.84 & 8.2 & 1970 \\
\hline GTN-1 & $15-20$ & 381 & 3.97 & 0.0378 & 0.0052 & 22.6 & 83.75 & 73 & 4730 \\
\hline GTN-1 & $20-25$ & 543 & 7.16 & 0.0446 & 0.0055 & 22.9 & 87.7 & 90 & 4740 \\
\hline GTN-2 & $0-5$ & 461 & 8.75 & 0.0364 & 0.0079 & 8.2 & 31.36 & 1.1 & 45.7 \\
\hline GTN-2 & $5-10$ & 457 & 3.64 & 0.0257 & 0.0048 & 18.1 & 32.44 & 2.4 & 66.4 \\
\hline GTN-2 & $10-15$ & 428 & 4.81 & 0.0271 & 0.0044 & 22 & 46.96 & 2 & 79.4 \\
\hline GTN-2 & $15-20$ & 456 & 6.8 & 0.0251 & 0.0039 & 24.7 & 62.6 & 2.2 & 152 \\
\hline GTN-2 & $20-25$ & 535 & 79.6 & 0.0221 & 0.004 & 19.4 & 71.37 & 2.5 & 111 \\
\hline GTN-3 & $0-5$ & 525 & 39.3 & 0.0376 & 0.009 & 8.8 & 131.3 & 2.8 & 1030 \\
\hline GTN-3 & $5-10$ & 705 & 11.5 & 0.022 & 0.0046 & 10.5 & 139.6 & 3 & 1110 \\
\hline GTN-3 & $10-15$ & 402 & 2.68 & 0.0163 & 0.0034 & 27 & 440.5 & 61 & 2050 \\
\hline GTN-4 & $0-5$ & 725 & 85.3 & 0.05 & 0.0089 & 7.03 & 67.68 & 8.5 & 3610 \\
\hline GTN-4 & $5-10$ & 1210 & 43.7 & 0.0879 & 0.0128 & 14 & 80.99 & 16 & 3850 \\
\hline GTN-4 & $10-15$ & 643 & 4.67 & 0.0168 & 0.0026 & 26.7 & 116.1 & 20 & 4280 \\
\hline GTN-4 & $15-20$ & 534 & 2.21 & 0.0167 & 0.0027 & 28.7 & 222.3 & 67 & 12500 \\
\hline GTN-4 & $20-25$ & 565 & 4.52 & 0.0131 & 0.0023 & 25.9 & 294.6 & 29 & 13500 \\
\hline KSN-1 & $0-5$ & 406 & 19.4 & 0.0063 & 0.0013 & 4.6 & 31.63 & 12 & 84.5 \\
\hline KSN-1 & $5-10$ & 559 & 38.2 & 0.0137 & 0.0035 & 7.44 & 24.27 & 5.2 & 58.3 \\
\hline KSN-1 & $10-15$ & 933 & 11.6 & 0.0339 & 0.0064 & 10 & 28.12 & 1.4 & 25.9 \\
\hline KSN-1 & $15-20$ & 534 & & 0.0323 & 0.0072 & 11 & 21.84 & 1.3 & 27.2 \\
\hline KSN-2 & $0-5$ & 253 & 17.8 & 0.0111 & 0.0025 & 4.85 & 48.74 & 27 & 13.4 \\
\hline KSN-2 & $5-10$ & 385 & 23.4 & 0.0312 & 0.006 & 4.71 & 57.55 & 1.5 & 9.9 \\
\hline KSN-2 & $10-15$ & 308 & 31.5 & 0.0335 & 0.0091 & 6.15 & 53.96 & 2.3 & 12.1 \\
\hline KSN-2 & $15-20$ & 464 & 24.3 & 0.0487 & 0.0099 & 4.53 & 39.21 & 1 & 12.5 \\
\hline KSN-2 & $20-25$ & 182 & 37.3 & 0.0243 & 0.0064 & 7.03 & 38.22 & 1.3 & 20.2 \\
\hline KSN-3 & $0-5$ & 202 & 20.9 & 0.0356 & 0.0084 & 4.18 & 34.11 & 2.2 & 20.6 \\
\hline KSN-3 & $5-10$ & 244 & 15.1 & 0.0373 & 0.0081 & 4.71 & 38.89 & 1.7 & 11.5 \\
\hline KSN-3 & $10-15$ & 296 & 21.3 & 0.0541 & 0.0111 & 4.97 & 45.96 & 2.7 & 11.4 \\
\hline KSN-3 & $15-20$ & 710 & 10.8 & 0.0125 & 0.0032 & 5.83 & 41.76 & 1.4 & 10 \\
\hline KSN-3 & $20-25$ & 823 & 1.92 & 0.0194 & 0.0042 & 10.6 & 39.12 & 1 & 9.5 \\
\hline KSN-4 & $0-5$ & 144 & 18.8 & 0.0166 & 0.0039 & 4.04 & 41.91 & 3.2 & 20 \\
\hline KSN-4 & $5-10$ & 259 & 19.7 & 0.0468 & 0.0104 & 4.39 & 43.46 & 2.1 & 18.3 \\
\hline KSN-4 & $10-15$ & 283 & 21.2 & 0.0708 & 0.014 & 5.97 & 38.98 & 1.7 & 16.2 \\
\hline KSN-4 & $15-20$ & 649 & 17 & 0.026 & 0.0061 & 11.3 & 39.59 & 1.2 & 12.3 \\
\hline KSN-4 & $20-25$ & 406 & 3.06 & 0.0111 & 0.0026 & 30.4 & 36.49 & 1.3 & 17.1 \\
\hline
\end{tabular}


Table S2. Characterisation of the boreal wetlands. SKM: Storkälsmyran, KSN: Kroksjön, LDN: Långedalen and GTN: Gästern. All data presented in this table were extracted from Liem et al., (2020).

\begin{tabular}{|c|c|c|c|c|c|c|c|c|c|c|c|c|c|c|c|c|c|c|}
\hline \multirow[b]{2}{*}{ No } & \multirow[t]{2}{*}{ pH } & \multirow{2}{*}{$\begin{array}{r}\mathrm{H}_{2} \mathrm{~S} \\
\mu \mathrm{M}\end{array}$} & \multirow{2}{*}{$\begin{array}{l}\mathbf{H g}^{\mathrm{II}} \\
\mathbf{p M}\end{array}$} & \multicolumn{3}{|c|}{$\begin{array}{c}\text { BiO Total } \\
\text { MeHgLMMLMM } \\
\text { thiols thiol }\end{array}$} & \multirow{2}{*}{$\begin{array}{c}\begin{array}{c}\text { RSH } \\
\text { (ads) }\end{array} \\
\mu \mathrm{mol} \mathrm{g}^{-1}\end{array}$} & \multirow{2}{*}{$\begin{array}{l}\mathrm{SO}_{4}{ }^{2-} \\
\mathrm{mg}^{\mathbf{L}^{-1}} \\
\end{array}$} & \multirow{2}{*}{$\begin{array}{l}\text { Total } \\
\mathbf{S} \\
\mathbf{m g} \\
\mathbf{L}^{-1} \\
\end{array}$} & \multirow{2}{*}{$\begin{array}{l}\mathrm{Cl}^{-} \\
\mathrm{mg}^{-1} \\
\mathrm{~L}^{-1} \\
\end{array}$} & \multirow{2}{*}{$\begin{array}{l}\mathbf{F e}^{\mathrm{II}} \\
\mu \mathrm{M}\end{array}$} & & C & $\mathbf{K}$ & Al & Mn & $\mathbf{P}$ & -NO3 \\
\hline & & & & $\mathbf{p M}$ & $\mathbf{n M}$ & $\mathbf{n M}$ & & & & & & & $\begin{array}{l}\mathbf{m g} \\
\mathbf{L}^{-1} \\
\end{array}$ & $\lg L^{-1}$ & $\begin{array}{l}\mathbf{m g} \\
\mathbf{L}^{-1} \\
\end{array}$ & 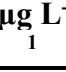 & 1 & $\mathrm{ug} / \mathrm{L}$ \\
\hline S & 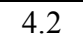 & 0.3 & & 4.9 & 5 & 48 & & 1.6 & 2.2 & 2.3 & 2 & 31 & 61 & r & 0.5 & 13 & 45 & 102 \\
\hline SKM-1 & 17 & 7.5 & 155 & 6.1 & 15 & 62 & 13 & 0.5 & 1.1 & 2.1 & 18 & 16 & 63 & .1 & 0.6 & 14 & 50 & 3.96 \\
\hline SKM-1 & 16 & 0.4 & 61 & 8.8 & 13 & 58 & & 0.4 & 1.1 & 1.9 & 18 & 25 & 68 & 0.1 & 0.7 & 14 & 38 & 5.92 \\
\hline SKM-1 & 4.21 & $<0.3$ & 126 & 9 & 3.5 & 20 & & 0.2 & 1 & 1.8 & 24 & 19 & 67 & 0.3 & 0.7 & 15 & 94 & 16.87 \\
\hline SKM-1 & 4.25 & 0.3 & 159 & 8.7 & 4.3 & 22 & & 0.2 & 0.9 & 1.8 & 36 & 0 & 68 & 0.1 & 0.7 & 15 & 82 & 17.63 \\
\hline SKM-2 & 4.46 & 0.3 & 40 & 17 & 8.1 & 40 & & $<0.1$ & 0.8 & 2.9 & 12 & 26 & 67 & 0.4 & 0.6 & 11 & 157 & 19.7 \\
\hline SKM & 4.61 & 4.9 & 129 & 14 & 3.9 & 26 & 15 & $<0.1$ & 0.8 & 2.2 & 21 & 15 & 68 & 0.6 & 0.6 & 10 & 316 & 20.21 \\
\hline SKM-2 & 4.66 & 14 & 48 & 16 & 3.2 & 29 & & 0.2 & 0.8 & 2.3 & 18 & 20 & 67 & 0.5 & 0.6 & 10 & 261 & 23.91 \\
\hline SKM-2 & 4.58 & 9.6 & 58 & 13 & 2.6 & 21 & & $<0.1$ & 0.9 & 1.6 & 36 & 9.4 & 74 & 0.7 & 0.8 & 11 & 426 & 20.91 \\
\hline SKM-2 & 4.72 & 11 & 109 & 26 & 3.9 & 22 & & $<0.1$ & 0.8 & 2 & 38 & 9.5 & 73 & 0.7 & 0.9 & 13 & 316 & 41.29 \\
\hline SKM- & 4.56 & $<0.3$ & 57 & 8.5 & 7.5 & 27 & 13 & $<0.1$ & 0.7 & 2.9 & 11 & 46 & 69 & & 0.7 & 18 & 76 & 11.69 \\
\hline SKM-3 & 4.8 & 2.5 & 38 & 18 & 5.1 & 34 & 6.9 & 0.5 & 1.3 & 2.3 & 14 & 62 & 70 & 0.1 & 0.9 & 22 & 33 & 17.66 \\
\hline SKM- & 4.91 & 0.7 & 108 & 9.1 & 9 & 54 & 4 & 0.4 & 1 & 1.2 & 13 & 73 & 52 & $<0.06$ & 0.6 & 22 & 30 & 9.45 \\
\hline SKM & 40 & 7.8 & 75 & 2.4 & 6.9 & 47 & & $<0.1$ & 0.8 & 0.5 & 16 & 100 & 53 & $<0.06$ & 0.5 & 27 & 48 & 3.13 \\
\hline SKM & 46 & 0.3 & 67 & 15 & 5.6 & 29 & & $<0.1$ & 0.7 & 2.8 & 10 & 49 & 71 & 0.3 & 0.7 & 10 & 38 & 0.04 \\
\hline SKM- & 4.67 & 0.3 & 70 & 11 & 6.5 & 29 & 9.5 & $<0.1$ & 0.7 & 2.9 & 11 & 5 & 0 & 0.5 & 0.6 & 7 & 40 & 17.51 \\
\hline SKM & 4.65 & $<0.3$ & 70 & 13 & 9.9 & 44 & & $<0.1$ & 0.8 & 2.8 & 14 & & & & 8 & 10 & 3 & 2.32 \\
\hline SKM & 56 & 51 & 76 & 9.1 & 7.8 & 25 & & $<0.1$ & 0.8 & 2.8 & 15 & 9 & & 4 & 0.9 & 12 & 100 & 22.65 \\
\hline SKM- & 4 & 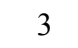 & 52 & 8.8 & 7.7 & 25 & & $<0.1$ & 0.8 & 2.7 & 17 & $7^{\prime}$ & 8 & 0.4 & 1 & 15 & 108 & 22.36 \\
\hline LDN & & $<0.3$ & 82 & & 6.9 & 25 & 3.9 & 4.1 & 4.6 & 8.2 & 1 & & & .4 & 1 & 179 & 34 & 13.08 \\
\hline LDN & & 44 & 44 & 72 & 21 & 61 & 6.4 & 3.6 & 4.3 & 8.4 & 4 & 15 & 7 & 7 & 1.2 & 167 & 51 & 0.38 \\
\hline LDN- & 4.4 & 19 & 59 & 92 & 37 & 102 & & 0.6 & 1.7 & 9.8 & 5 & 7 & 7 & 0.7 & + & 142 & 70 & 10.72 \\
\hline LDN- & 450 & 51 & 41 & & 11 & 71 & & 0.1 & 1.2 & 9.6 & 4 & & & 2 & 1.1 & 110 & 7 & 16.61 \\
\hline LD] & & 34 & 23 & 74 & 8.5 & 47 & 0 & $<0.1$ & 1.2 & 9.1 & 40 & 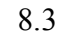 & - & 1 & 0.9 & 97 & 45 & 13.23 \\
\hline LDN- & 5.64 & 0.3 & 77 & 6.8 & 3.2 & 13 & & 0.3 & 0.8 & 9.7 & 2.7 & 25 & 4 & 0.6 & 0.5 & 99 & $<20$ & 17.93 \\
\hline LDN- & 5.87 & 0.3 & 41 & 3 & 3.5 & & & 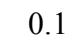 & 0.7 & 8.8 & 8 & & & 6 & 5 & 197 & $<20$ & 36.86 \\
\hline LDN & & .3 & 38 & 3.2 & 2.9 & 20 & & 01 & 0 & 8.8 & 6.4 & 5 & 3 & 6 & 0.4 & 183 & $<20$ & 26.3 \\
\hline LDN- & 5.89 & $<0.3$ & 30 & 2.7 & 3 & 16 & & 0 . & 0.6 & 8.3 & 9 & 6 & 4 & 0.6 & 0.5 & $<1$ & $<20$ & 13.09 \\
\hline LDN- & & 1.1 & 68 & 21 & 4.5 & 11 & & 0.4 & 0.9 & 7.7 & 3 & & & 3 & 0.9 & $<1$ & 25 & 29.36 \\
\hline LDN & & 1 & 138 & 8.6 & 0.9 & 4.6 & & 0.3 & 0.9 & 8.4 & 4 & & 34 & $<0.06$ & 1 & 170 & 21 & 35.75 \\
\hline LDN- & 5.6 & $<0.3$ & 101 & 5.1 & 3.1 & 12 & & 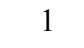 & 1.2 & 7.2 & & 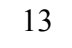 & 1 & - & 0.2 & 219 & $<20$ & 16.5 \\
\hline LDN & 60 & 0.4 & 221 & 4.8 & 2.4 & 8.5 & 6.3 & 1 & 1.2 & 7 & 0.1 & 16 & 1 & 0.9 & 0.2 & 208 & $<20$ & 13.98 \\
\hline LDN & & 0.3 & 35 & 5.8 & 2.5 & 9.8 & & 0.9 & 1.1 & 7 & 0.1 & ? & & & 0.1 & $<1$ & $<20$ & 15.68 \\
\hline LDN- & - & $<0.3$ & 145 & 5.3 & 2. & 1.5 & & $<0.1$ & 0. & 5.9 & 352 & . & 20 & & 1 & $<1$ & $<20$ & 22.7 \\
\hline LDN & & 17 & 45 & 43 & 1.1 & 5.5 & & $<0.1$ & 0.3 & 7.1 & 10 & 220 & 26 & 4 & 0.2 & $<1$ & $<20$ & 36.92 \\
\hline LDN & & $<0.3$ & 74 & .7 & 3.1 & 14 & 4 & $<0.1$ & 0.6 & 5.9 & 4.5 & 2 & & 2 & 0.4 & 98 & 53 & 13.99 \\
\hline LDN- & & $<0.3$ & 246 & & 5.4 & 2 & & & 0.9 & 7.6 & & 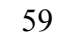 & 8 & .7 & 0.9 & 119 & 52 & 17.17 \\
\hline $\mathrm{LDN}$ & 5.02 & 0.4 & 216 & 3.3 & 2.6 & 15 & 6 & 0.1 & 0.9 & 7 & 4.9 & 61 & . & 3 & 0.7 & 132 & 60 & 43.88 \\
\hline LDN- & & 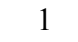 & 179 & 9.5 & 6.9 & 22 & & $<0.1$ & 1.2 & 6.2 & 23 & 82 & 12 & .1 & 0.8 & 90 & 89 & 42.77 \\
\hline LDN- & 4. & 0 & 15 & 5.5 & 3.6 & - & & $<0.1$ & 1.9 & 5.9 & 00 & 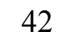 & 236 & 0.1 & 1.3 & 55 & 123 & 55.45 \\
\hline GTN- & 5 & 21 & 59 & 3.9 & 3.1 & 20 & & 1.3 & 2.3 & 12 & 4.1 & 52 & 49 & 1.6 & 0.3 & 57 & 51 & 42.06 \\
\hline GTN- & 5.6 & 1 & 50 & 4.4 & 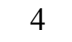 & 17 & & 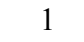 & 2.2 & 12 & 4 & ? & - & 1.8 & 0.7 & 71 & 53 & 11.68 \\
\hline GTN- & & $2 ?$ & 30 & 3.6 & 7.1 & 28 & 0.0 & 1 & 2.3 & 12 & 5.8 & נו & 62 & 1.8 & 0.8 & 82 & 86 & 10.78 \\
\hline GTN- & 5.62 & 13 & 52 & 3.8 & 4.2 & 26 & 7.6 & 0.2 & 2 & 11 & 17 & 114 & 84 & 3.2 & 1.1 & 50 & 327 & 13.61 \\
\hline GTN- & 5.5 & 13 & 261 & 2.8 & 4.3 & 24 & 7.7 & $<0.1$ & 2 & 11 & 20 & 123 & 8 & 3.2 & 1.1 & 21 & 374 & 12.07 \\
\hline GTN-2 & & 270 & 23 & 4.1 & 3.9 & 26 & & 3.9 & 4.6 & 14 & 1.5 & 11 & 31 & 0.4 & 0.3 & 71 & $<20$ & 7.95 \\
\hline GTN-2 & 5.86 & 190 & 58 & 2.9 & 3.4 & 15 & & 2.6 & 3.4 & 14 & 2.4 & 17 & 32 & 0.5 & 0.3 & 77 & 24 & 44.23 \\
\hline GTN- & & 4 & 37 & 1.8 & 2.3 & 7.8 & & & 1 & 15 & 26 & & 4 & 0.1 & 0.4 & 98 & 32 & 31.32 \\
\hline GTN-2 & 6.06 & 180 & 31 & 3.1 & 5.3 & 22 & & 0.2 & 2.2 & 13 & 90 & 124 & 63 & 0.1 & 0.5 & 116 & 54 & 31.37 \\
\hline GTN-2 & 5.86 & 120 & 31 & 4.2 & 5.1 & 29 & & 0.1 & 2.6 & 13 & 165 & 112 & 71 & 0.1 & 0.9 & 120 & 106 & 33.91 \\
\hline GTN-3 & 4.79 & 0.3 & 47 & 6.9 & 4.5 & 23 & & 1.2 & 4.9 & 7.1 & 18 & 60 & 13 & 6.3 & 2.1 & 139 & 130 & 65.23 \\
\hline GTN-3 & 4.5 & $<0.3$ & 164 & 4.5 & 4.6 & 28 & & 1.2 & 5 & 7.5 & 22 & 08 & 140 & .0 & 2.1 & 145 & 132 & 63.75 \\
\hline GTN-3 & 4.88 & $<0.3$ & 556 & 7.6 & 4.1 & 45 & & 0.3 & 11 & 13 & 375 & 269 & 440 & 1.3 & 3.8 & $<1$ & 458 & 52.52 \\
\hline GTN- & & 13 & 31 & 11 & 5.5 & 32 & & 0.9 & 3.6 & 9.9 & 5 & 159 & 68 & 0.9 & 0.6 & 59 & 69 & 46.41 \\
\hline GTN-4 & 6.08 & 17 & 31 & 16 & 14 & 38 & & 0.6 & 4.1 & 9.4 & 47 & 150 & 81 & 0.7 & 0.9 & 79 & 106 & 54.96 \\
\hline GTN-4 & 6.09 & 10 & 140 & 16 & 3.5 & 13 & & 0.3 & 4.9 & 9 & 164 & 138 & 116 & 0.6 & 1.2 & 91 & 171 & 36.95 \\
\hline GTN-4 & 5.97 & 10 & 442 & 16 & 2.1 & 33 & & $<0.1$ & 7.9 & 7.2 & 172 & 183 & 222 & 1.6 & 2.9 & 53 & 300 & 34.8 \\
\hline
\end{tabular}




\begin{tabular}{lcccccccccccccccccc} 
GTN-4 & 5.93 & 27 & 217 & 6.7 & 1.7 & 23 & & $<0.1$ & 12 & 9 & 317 & 109 & 295 & 2.1 & 4.5 & 68 & 305 & 68.6 \\
KSN-1 & 4.64 & $<0.3$ & 23 & 3.2 & 4.1 & 27 & & 0.2 & 0.5 & 1.2 & 1.1 & 24 & 32 & 1.5 & 0.2 & 2 & 65 & 44.12 \\
KSN-1 & 4.45 & $<0.3$ & 17 & 3.5 & 3.3 & 21 & 9.4 & $<0.1$ & 0.3 & 0.9 & 0.7 & 36 & 24 & 0.5 & 0.3 & 4 & 32 & 39.36 \\
KSN-1 & 4.73 & $<0.3$ & 4.4 & 4.2 & 0.2 & 36 & & $<0.1$ & 0.4 & 0.6 & 1.7 & 78 & 28 & 0.2 & 0.5 & 24 & 38 & 40.83 \\
KSN-1 & 4.81 & $<0.3$ & 2.2 & 3.6 & 4.7 & 63 & & $<0.1$ & 0.3 & 0.5 & 0.2 & 63 & 22 & 0.2 & 0.4 & 21 & 29 & 45.7 \\
KSN-2 & 4.65 & $<0.3$ & 34 & 5.2 & 3.6 & 8.8 & & 0.3 & 0.8 & 3 & 3.6 & 44 & 49 & 0.9 & 0.6 & 3 & 113 & 25.46 \\
KSN-2 & 4.81 & $<0.3$ & 30 & 6.7 & 3.9 & 8.4 & 6.6 & 0.1 & 0.8 & 2.6 & 7.4 & 81 & 58 & 0.2 & 0.9 & 6 & 56 & 23.48 \\
KSN-2 & 4.85 & $<0.3$ & 22 & 4 & 0.5 & 18 & & 0.1 & 0.7 & 2.5 & 6.6 & 102 & 54 & 0.3 & 0.8 & 10 & 54 & 28.43 \\
KSN-2 & 5.36 & $<0.3$ & 30 & 2.8 & 1.9 & 24 & & $<0.1$ & 0.5 & 2.1 & 4.5 & 247 & 39 & 0.1 & 0.6 & 30 & 45 & 34.23 \\
KSN-2 & 5.82 & $<0.3$ & 17 & 1.8 & 1.6 & 7.1 & & $<0.1$ & 0.5 & 2.3 & 129 & 178 & 38 & 0.6 & 0.7 & 59 & 53 & 35.16 \\
KSN-3 & 4.62 & 0.4 & 10 & 3.2 & 3.3 & 48 & 5.3 & $<0.1$ & 0.4 & 2.2 & 0.1 & 24 & 34 & 0.5 & 0.5 & 8 & $<20$ & 42.02 \\
KSN-3 & 5.54 & 1.4 & 40 & 3.2 & 3.3 & 64 & 9.9 & $<0.1$ & 0.5 & 2.2 & 0.1 & 28 & 39 & 0.6 & 0.5 & 8 & 23 & 31.37 \\
KSN-3 & 4.77 & 4 & 25 & 2.7 & 2.7 & 49 & 22 & $<0.1$ & 0.6 & 1.4 & 1.1 & 71 & 46 & 0.3 & 0.9 & 14 & 38 & 27.77 \\
KSN-3 & 5.04 & 6.8 & 19 & 1.9 & 1.8 & 29 & 22 & $<0.1$ & 0.6 & 1.2 & 2.2 & 135 & 42 & 0.2 & 1.1 & 27 & 26 & 22.56 \\
KSN-3 & 5.42 & 4.8 & 19 & 1.8 & 2 & 22 & 11 & $<0.1$ & 0.5 & 1.3 & 1.5 & 145 & 39 & 0.3 & 1.1 & 30 & 27 & 22.76 \\
KSN-4 & 4.6 & $<0.3$ & 19 & 3.2 & 3.6 & 45 & & $<0.1$ & 0.5 & 3.5 & 0.1 & 22 & 42 & 1.6 & 0.3 & 4 & 38 & 46.27 \\
KSN-4 & 4.7 & $<0.3$ & 31 & 3.9 & 3.5 & 119 & 10 & $<0.1$ & 0.5 & 3.2 & 0.7 & 33 & 43 & 0.9 & 0.4 & 6 & 35 & 30.37 \\
KSN-4 & 4.96 & $<0.3$ & 13 & 2.9 & 2.4 & 126 & & $<0.1$ & 0.7 & 3 & 1.7 & 93 & 39 & 0.4 & 0.4 & 26 & 33 & 30.59 \\
KSN-4 & 5.3 & $<0.3$ & 41 & 1.4 & 2.3 & 44 & & $<0.1$ & 0.7 & 3 & 17 & 140 & 40 & 0.3 & 0.5 & 37 & 40 & 29.21 \\
KSN-4 & 5.32 & $<0.3$ & 29 & 0.7 & 0.6 & 47 & & $<0.1$ & 0.6 & 2.8 & 136 & 122 & 36 & 0.6 & 0.8 & 64 & 48 & 37.8 \\
\hline
\end{tabular}


Table S3. Relative hgcA sequence abundances (\%) at levels of phyla, class, order and family for the combined 81-sample dataset that included all four wetlands. Taxonomic levels are indicated with stepwise indents. Mean \pm SD: standard deviation.

\begin{tabular}{|c|c|c|c|c|c|c|}
\hline \multirow[b]{2}{*}{ phylum } & \multirow[b]{2}{*}{ class } & \multirow[b]{2}{*}{ order } & \multicolumn{2}{|c|}{ Northern } & \multicolumn{2}{|c|}{ Southern } \\
\hline & & & SKM & $\mathrm{KSN}$ & GTN & LDN \\
\hline & & & mean std & mean std & mean std & mean std \\
\hline Archaea & & & $0.30 \pm 0.54$ & $3.38 \pm 3.67$ & $3.63 \pm 4.65$ & $3.26 \pm 3.91$ \\
\hline Halobacterota & Methanosarcinia & Methanosarcinales & $0.28 \pm 0.48$ & $3.33 \pm 3.47$ & $3.22 \pm 3.05$ & $3.24 \pm 3.85$ \\
\hline \multirow[t]{2}{*}{ Thermoplasmatota } & Thermoplasmata & Methanomassiliicoccales & $0.02 \pm 0.05$ & $0.01 \pm 0.04$ & $0.01 \pm 0.01$ & $0.02 \pm 0.05$ \\
\hline & unclass & unclass & $0.00 \pm 0.00$ & $0.04 \pm 0.17$ & $0.40 \pm 1.59$ & $0.00 \pm 0.00$ \\
\hline \multicolumn{7}{|l|}{ Bacteria } \\
\hline Unclass & unclass & Bact_unclass & $51.78 \pm 22.05$ & $32 \pm 18.5$ & $21.2 \pm 17.86$ & $34.1 \pm 20.7$ \\
\hline \multirow[t]{3}{*}{ Acidobacteriota } & & & $0.84 \pm 1.61$ & $0.32 \pm 0.31$ & $0.19 \pm 0.20$ & $0.79 \pm 1.17$ \\
\hline & Acidobacteriae & Bryobacterales & $0.05 \pm 0.08$ & $0.01 \pm 0.02$ & $0.00 \pm 0.00$ & $0.00 \pm 0.00$ \\
\hline & UBA6911 & unclass & $0.79 \pm 1.54$ & $0.31 \pm 0.30$ & $0.19 \pm 0.20$ & $0.79 \pm 1.17$ \\
\hline \multirow[t]{3}{*}{ Actinobacteriota } & & & $5.29 \pm 14$ & $21.9 \pm 33$ & $9.43 \pm 13$ & $1.99 \pm 4$ \\
\hline & Coriobacteriia & OPB41 & $4.43 \pm 11.16$ & $18.08 \pm 18.17$ & $8.78 \pm 11.47$ & $0.40 \pm 0.37$ \\
\hline & unclass & unclass & $0.86 \pm 2.84$ & $3.86 \pm 14.50$ & $0.65 \pm 1.32$ & $1.59 \pm 3.15$ \\
\hline \multirow[t]{3}{*}{ Bacteroidota } & & & $1.12 \pm 2$ & $0.94 \pm 1$ & $2.16 \pm 2$ & $3.57 \pm 6$ \\
\hline & Bacteroidia & Bacteroidales & $0.01 \pm 0.02$ & $0.32 \pm 0.52$ & $0.04 \pm 0.12$ & $0.10 \pm 0.49$ \\
\hline & UBA10030 & UBA10030 & $1.12 \pm 2.01$ & $0.63 \pm 0.78$ & $2.11 \pm 2.06$ & $3.46 \pm 5.02$ \\
\hline \multirow[t]{3}{*}{ Chloroflexota } & & & $0.01 \pm 0.05$ & $0.00 \pm 0.01$ & $0.00 \pm 0.00$ & $0.00 \pm 0.01$ \\
\hline & Anaerolineae & Anaerolineales & $0.00 \pm 0.00$ & $0.00 \pm 0.01$ & $0.00 \pm 0.00$ & $0.00 \pm 0.01$ \\
\hline & Dehalococcoidia & Dehalococcoidales & $0.01 \pm 0.05$ & $0.00 \pm 0.00$ & $0.00 \pm 0.00$ & $0.00 \pm 0.00$ \\
\hline \multirow[t]{9}{*}{ Desulfobacterota } & & & $1.97 \pm 3.19$ & $0.98 \pm 1.63$ & $0.23 \pm 0.49$ & $1.57 \pm 3.96$ \\
\hline & BSN033 & SM23-61 & $0.01 \pm 0.04$ & $0.00 \pm 0.00$ & $0.00 \pm 0.00$ & $0.00 \pm 0.00$ \\
\hline & Desulfobacteria & Desulfobacterales & $0.00 \pm 0.00$ & $0.00 \pm 0.00$ & $0.00 \pm 0.01$ & $0.00 \pm 0.01$ \\
\hline & Desulfobulbia & Desulfobulbales & $0.00 \pm 0.00$ & $0.00 \pm 0.00$ & $0.00 \pm 0.01$ & $0.66 \pm 2.04$ \\
\hline & Desulfobulbia & unclass & $0.01 \pm 0.03$ & $0.20 \pm 0.42$ & $0.04 \pm 0.07$ & $0.24 \pm 0.80$ \\
\hline & Desulfomonilia & UBA1062 & $0.00 \pm 0.00$ & $0.00 \pm 0.00$ & $0.01 \pm 0.02$ & $0.01 \pm 0.03$ \\
\hline & Syntrophia & Syntrophales & $1.39 \pm 1.99$ & $0.26 \pm 0.35$ & $0.05 \pm 0.05$ & $0.45 \pm 0.70$ \\
\hline & Syntrophorhabdia & Syntrophorhabdales & $0.42 \pm 0.83$ & $0.05 \pm 0.15$ & $0.12 \pm 0.32$ & $0.03 \pm 0.05$ \\
\hline & unclass & unclass & $0.13 \pm 0.29$ & $0.47 \pm 0.71$ & $0.01 \pm 0.03$ & $0.18 \pm 0.34$ \\
\hline \multirow[t]{2}{*}{ Desulfobacterota_A } & & & $8.91 \pm 11.55$ & $3.19 \pm 5.65$ & $6.85 \pm 12.46$ & $0.99 \pm 1.17$ \\
\hline & Desulfovibrionia & Desulfovibrionales & $8.91 \pm 11.55$ & $3.19 \pm 5.65$ & $6.85 \pm 12.46$ & $0.99 \pm 1.17$ \\
\hline \multirow[t]{3}{*}{ Desulfuromonadota } & & & $3.86 \pm 2.72$ & $15.44 \pm 14.12$ & $23.31 \pm 18.74$ & $12.9 \pm 11.82$ \\
\hline & Desulfuromonadia & Desulfuromonadales & $3.84 \pm 2.67$ & $15.43 \pm 14.10$ & $23.31 \pm 18.74$ & $12.89 \pm 11.81$ \\
\hline & & unclass & $0.02 \pm 0.05$ & $0.00 \pm 0.00$ & $0.00 \pm 0.00$ & $0.00 \pm 0.00$ \\
\hline \multirow[t]{3}{*}{ Elusimicrobiota } & & & $0.58 \pm 1.53$ & $1.22 \pm 2.03$ & $0.64 \pm 1.61$ & $0.03 \pm 0.06$ \\
\hline & Elusimicrobia & Elusimicrobiales & $0.58 \pm 1.53$ & $1.22 \pm 2.03$ & $0.64 \pm 1.61$ & $0.03 \pm 0.05$ \\
\hline & Endomicrobia & unclass & $0.00 \pm 0.00$ & $0.00 \pm 0.00$ & $0.00 \pm 0.00$ & $0.00 \pm 0.01$ \\
\hline \multirow[t]{2}{*}{ Fibrobacterota } & & & $0.79 \pm 1.69$ & $0.19 \pm 0.49$ & $0.17 \pm 0.30$ & $0.40 \pm 0.63$ \\
\hline & OXYB2-FULL-49-7 & OXYB2-FULL-49-7 & $0.13 \pm 0.24$ & $0.13 \pm 0.38$ & $0.05 \pm 0.09$ & $0.13 \pm 0.37$ \\
\hline
\end{tabular}




\begin{tabular}{|c|c|c|c|c|c|c|}
\hline & unclass & unclass & $0.65 \pm 1.46$ & $0.06 \pm 0.11$ & $0.12 \pm 0.21$ & $0.27 \pm 0.27$ \\
\hline \multirow[t]{3}{*}{ Firmicutes_A } & & & $0.02 \pm 0.09$ & $0.02 \pm 0.04$ & $0.00 \pm 0.01$ & $0.01 \pm 0.03$ \\
\hline & Clostridia & Clostridiales & $0.00 \pm 0.00$ & $0.00 \pm 0.00$ & $0.00 \pm 0.00$ & $0.00 \pm 0.01$ \\
\hline & Clostridia & Peptostreptococcales & $0.02 \pm 0.09$ & $0.02 \pm 0.04$ & $0.00 \pm 0.01$ & $0.00 \pm 0.02$ \\
\hline \multirow[t]{4}{*}{ Firmicutes_B } & & & $2.11 \pm 4.56$ & $1.09 \pm 1.71$ & $1.16 \pm 2.79$ & $0.63 \pm 1.60$ \\
\hline & Desulfitobacteriia & Desulfitobacteriales & $0.88 \pm 2.87$ & $0.00 \pm 0.01$ & $0.00 \pm 0.00$ & $0.01 \pm 0.03$ \\
\hline & Desulfotomaculia & Desulfotomaculales & $0.02 \pm 0.07$ & $0.01 \pm 0.04$ & $0.00 \pm 0.00$ & $0.10 \pm 0.49$ \\
\hline & Syntrophomonadia & Syntrophomonadales & $1.21 \pm 1.62$ & $1.08 \pm 1.66$ & $1.16 \pm 2.79$ & $0.52 \pm 1.08$ \\
\hline Firmicutes_C & Negativicutes & unclass & $0.00 \pm 0.01$ & $0.00 \pm 0.00$ & $0.00 \pm 0.00$ & $0.00 \pm 0.00$ \\
\hline Goldbacteria & PGYV01 & unclass & $\mathbf{0 . 0 0} \pm \mathbf{0 . 0 0}$ & $0.00 \pm 0.00$ & $0.02 \pm 0.04$ & $0.00 \pm 0.00$ \\
\hline Margulisbacteria & WOR-1 & O2-12-FULL-45-9 & $0.09 \pm 0.19$ & $0.00 \pm 0.01$ & $0.00 \pm 0.00$ & $0.00 \pm 0.00$ \\
\hline Myxococcota & Polyangia & Palsa-1104 & $0.06 \pm 0.19$ & $0.08 \pm 0.19$ & $0.00 \pm 0.00$ & $0.01 \pm 0.02$ \\
\hline Nitrospirota & Thermodesulfovibrionia & Thermodesulfovibrionales & $0.68 \pm 0.48$ & $0.36 \pm 0.45$ & $0.55 \pm 0.85$ & $0.31 \pm 0.45$ \\
\hline \multirow[t]{3}{*}{ Spirochaetota } & & & $1.50 \pm 1.79$ & $0.14 \pm 0.18$ & $0.06 \pm 0.14$ & $0.18 \pm 0.20$ \\
\hline & Spirochaetia & Spirochaetales & $1.50 \pm 1.79$ & $0.14 \pm 0.18$ & $0.05 \pm 0.13$ & $0.18 \pm 0.19$ \\
\hline & UBA6919 & GWB1-36-13 & $0.00 \pm 0.00$ & $0.00 \pm 0.00$ & $0.00 \pm 0.01$ & $0.00 \pm 0.01$ \\
\hline Synergistota & Synergistia & Synergistales & $0.00 \pm 0.00$ & $0.00 \pm 0.02$ & $0.00 \pm 0.00$ & $0.00 \pm 0.00$ \\
\hline \multirow[t]{3}{*}{ Verrucomicrobiota } & & & $0.07 \pm 0.12$ & $0.00 \pm 0.02$ & $0.00 \pm 0.01$ & $0.04 \pm 0.13$ \\
\hline & Kiritimatiellae & LD1-PB3 & $0.00 \pm 0.00$ & $0.00 \pm 0.01$ & $0.00 \pm 0.00$ & $0.00 \pm 0.01$ \\
\hline & Lentisphaeria & unclass & $0.07 \pm 0.12$ & $0.00 \pm 0.01$ & $0.00 \pm 0.01$ & $0.04 \pm 0.12$ \\
\hline WOR-3 & WOR-3 & unclass & $1.00 \pm 2.88$ & $0.42 \pm 0.60$ & $0.11 \pm 0.15$ & $0.45 \pm 1.64$ \\
\hline Unclass & unclass & unclass & $19.01 \pm 13.71$ & $18.27 \pm 11.42$ & $30.25 \pm 13.78$ & $38.8 \pm 15.51$ \\
\hline
\end{tabular}

